\title{
Contenido y distribución de macronutrientes en caléndula (Caléndula officinalis L.) en el Valle del Cauca, Colombia
}

\section{Content and distribution of macronutrients in calendula (Calendula officinalis I.) in the Valle del Cauca, Colombia}

Conteúdo e distribuição de macronutrientes na Calendula (Calendula officinalis L.), no Valle del Cauca, Colômbia

\author{
Yuly Samanta García Vivas ${ }^{1}$, Juan Carlos Menjivar Flores², \\ Manuel Salvador Sánchez Orozco ${ }^{3}$ \& Carmen Rosa Bonilla Correa ${ }^{4}$ \\ IIngeneira Agrónoma, Magister en Ciencias Agrarias. ${ }^{2}$ Ingeniero Agrónomo, Magister en Suelos y \\ Aguas, Doctor en Ciencia del Suelo. ${ }^{3}$ Ingeniero Agrónomo, Magister en Sistemas de Semillas. \\ ${ }^{4}$ Ingeniera Agrónoma, Magister en Suelos y Aguas. \\ ${ }^{1}$ Departamento de Suelos. Centro universitario del Litoral Atlántico. \\ Universidad Nacional Autónoma de Honduras. 2,3,4Facultad de Ciencias \\ Agropecuarias. Universidad Nacional de Colombia. Sede Palmira \\ 1ysgarciav@unal.edu.co, ${ }^{1} \mathrm{jcm}$ enjivar@unal.edu.co, ${ }^{3} \mathrm{mssanchezo@unal.edu.co,}$ \\ ${ }^{4}$ crbonillac@unal.edu.co
}

\section{Resumen}

Con el objetivo de evaluar el contenido y distribución de macronutrientes ( $\mathrm{N}, \mathrm{P}, \mathrm{K})$ en tallos, hojas, flores y semillas de caléndula (Calendula officinalis $\mathrm{L}$.) en diferentes épocas de desarrollo se realizó el presente estudio en el municipio de Yumbo, departamento del Valle del Cauca, Colombia. Se estableció en un diseño completamente al azar un experimento con cinco tratamientos (muestreos a los 2, 3, 5, 6 y 7 meses después del trasplante (mdt)) con tres repeticiones, se analizó en los órganos vegetales el contenido de N, P y K en cada etapa de crecimiento. El análisis de varianza mostró diferencias significativas en los contenidos de $\mathrm{N}, \mathrm{P}$ y K entre épocas y entre órganos vegetales. El nutriente con mayor demanda en todo el ciclo fue el $\mathrm{K}\left(197,40 \mathrm{~g} \mathrm{~kg}^{-1}\right)$ seguido del $\mathrm{N}\left(130,53 \mathrm{~g} \mathrm{~kg}^{-1}\right)$ y $\mathrm{P}\left(21,19 \mathrm{~g} \mathrm{~kg}^{-1}\right)$; los mayores contenidos de $\mathrm{N}$ y $\mathrm{P}$ se localizaron en las flores y en los tallos el K. La mayor demanda $\mathrm{P}$ y $\mathrm{K}$ se registró en el segundo y tercer mdt y en el tercer y séptimo mdt se registraron las mayores demandas de $\mathrm{N}$.

Palabras clave: Asterácea, capítulo floral, etapa fenológica, nutrición mineral.

\section{Abstract}

In order to evaluate the content and distribution of macronutrients $(\mathrm{N}, \mathrm{P}, \mathrm{K})$ in stems, leaves, flower heads and seeds of calendula (Calendula officinalis $\mathrm{L}$.) in different periods of development a study in the municipality of Yumbo, department of Valle del Cauca, Colombia was conducted. An experiment with five treatments (sampling at 2, 3, 5, 6 and 7 months after transplantation) with three replications in a completely randomized design was established; the content of N, P and K was analyzed 
in each plant organ and stage of growth. Analysis of variance showed significant differences in contents of $\mathrm{N}, \mathrm{P}$ and $\mathrm{K}$ among periods and plant organs. The nutrient with highest demand throughout the cycle was $\mathrm{K}\left(197,40 \mathrm{~g} \mathrm{~kg}^{-1}\right)$ followed by $\mathrm{N}$ $\left(130,53 \mathrm{~g} \mathrm{~kg}^{-1}\right)$ and $\mathrm{P}\left(21,19 \mathrm{~g} \mathrm{~kg}^{-1}\right)$; the highest contents of $\mathrm{N}$ and $\mathrm{P}$ were located on the flower heads ; whereas for $\mathrm{K}$, in the stems. The highest demand of $\mathrm{P}$ and $\mathrm{K}$ was observed in the second and third sampling. During the third and seventh sampling the highest demands for $\mathrm{N}$ were observed.

Key-words: Asteracea, flower head, mineral nutrition, phenological stage

\section{Resumo}

Visando avaliar o conteúdo de distribuição elementos maiores ( $N, P, K)$ em caules, folhas, flores e sementes de calêndula (Calendula officinalis L.) em épocas diferentes de desenvolvimento, foi realizado o presente estudo no município de Yumbo, estado do Vale do Cauca, Colômbia. Foi estabelecido em um delineamento inteiramente casualizado um experimento com cinco tratamentos (amostragem a 2, 3, 5, 6 y 7 meses despois do trasplante (mdt)) com três repetições, analisando nos órgãos vegetais 0 conteúdo de N, P y K em cada faz de crescimento. A análise de variância mostrou diferencias significativas nos conteúdos de $\mathrm{N}, \mathrm{P}$ y $\mathrm{K}$ entre épocas e órgãos vegetais. O nutrimento com maior demanda em todo o ciclo foi K (197,40 g kg-1) seguido de N (130,53 g kg-1) e P (21,19 g kg-1), sendo que os maiores conteúdos de $\mathrm{N}$ e $\mathrm{P}$ encontraram-se nas flores, enquanto que nos caules o maior conteúdo foi de $\mathrm{K}$. A maior demanda de $\mathrm{P}$ e $\mathrm{K}$ se registrou no segundo e tercer mdt enquanto que no terceiro e septimo mdt se registraram as maiores demandas de $\mathrm{N}$.

Palavras-chave: Asteraceae, capítulo floral, estádio fenológico, nutrição mineral.

\section{Introducción}

La importancia de las plantas medicinales es reconocida y suficientemente documentada desde épocas ancestrales; en la actualidad, el Estado colombiano basado en estudios de prospección y de mercado realizados por el Ministerio de Comercio, Industria y Turismo, Programa Transformación Productiva, ha confirmado que las tendencias del mercado mundial en el sector de cosméticos, aseo y limpieza, favorecen las ventajas comparativas del país, ya que este sector está demandando ingredientes naturales (Mincomex, 2009).

A pesar de que la caléndula es una especie introducida, se ha adaptado muy bien a las condiciones edafoclimáticas de varios países, incluyendo a Colombia, con buenos rendimientos y mercados (Conpes 3514, 2008). Esta especie es la más utilizada entre las 156 especies de plantas medicinales, aromáticas y condimentarías que se comercializan en Colombia (IGAC, 2006) y la segunda en el número de autorizaciones de comercialización (Guevara, Luengas \& Garavito, 2010).

La caléndula ( $C$. officinalis L.) pertenece a la familia Asteraceae (Pérez, 1956; García, 1975; Sánchez, 1980); la planta completa y sus flores poseen múltiples usos tradicionales y actividades farmacológicas, como: antiflogísticas, antisépticas, antiespasmódicas, astringentes, diaforética, antibacteriana, cicatrizante, antiinflamatoria, emenagoga e inmuno-moduladora. Además, se emplea en casos de acné, contusiones, golpes, torceduras, eczemas, quemaduras, picaduras de insectos, irritaciones cutáneas, amenorrea y dismenorrea (Cáceres, 1996; Acosta, Rodríguez \& Sánchez, 2001).

Los sistemas de producción agrícola requieren el conocimiento de la importancia que los nutrientes tienen para las plantas, ya que permiten la realización de procesos vitales relacionados 
con la absorción, transporte y utilización de asimilados, siendo estos elementos de naturaleza inorgánica o mineral (Castro \& Gómez, 2010). Alarcón (2000) afirma que la fertilidad del suelo se mantiene cuando las pérdidas son compensadas por las aportaciones, así, el manejo debe promover un balance armónico entre las pérdidas y aportes de nutrientes.

Los estudios sobre la nutrición mineral de plantas medicinales son relativamente escasos en Brasil, Fernandes et al. (2013) evaluaron el efecto de la aplicación de $100 \mathrm{~kg}$ de $\mathrm{N}$ y 50 de $\mathrm{P}$ en forma de úrea, bovinaza y mulch (Paspalum notatum) sin encontrar variaciones estadísticas $(p>0.05)$ en los análisis cromatográficos de los metabolitos secundarios identificados en las diferentes épocas de cosecha y en los tratamientos de fertilización evaluados en caléndula, resultados similares a los reportados por Borella et al. (2011). Sin embargo, estos resultados fueron contrastantes con los obtenidos por Araujo et al. (2009) y Paim et al. (2010) quienes reportaron que la fertilización nitrogenada incrementa el contenido total de flavonoides, y Król (2011) reportó el incremento del número de inflorescencias y de aceites esenciales en el cultivar Orange King de caléndula. Moreira et al (2005) lograron la máxima producción de materia fresca de caléndula $(20,52 \mathrm{~g}$ planta $^{-1}$ ) con la aplicación de 210,62 g planta $^{-1}$ de $\mathrm{N}$ en condiciones de invernadero.

Gonçalves et al (2012) evaluaron la disponibilidad de nutrientes y metales pesados tóxicos presentes en caléndula ( $C$. officinalis L.) en un experimento con aplicación de fertilización (orgánica e inorgánica) en tres niveles y dos texturas de suelo (arenoso y arcilloso): los resultados mostraron que el suelo arcilloso promovió la disponibilidad de N, P, K, Mg, Cu, Zn y Fe, por otro parte, el suelo arenoso favoreció la disponibilidad de $\mathrm{Ca}, \mathrm{Mn}, \mathrm{Pb}$ y $\mathrm{Cr}$, siendo evidente que el tejido de las hojas de plantas fertilizadas inorgánicamente presentó mayores concentraciones de $\mathrm{K}$ y $\mathrm{Mn}$.
En Colombia, aunque la caléndula es un cultivo importante, los estudios en nutrición vegetal son escasos y los productores desconocen el momento en el que se requiere mayor concentración de nutrientes en la planta y que pueda ser beneficioso para los consumidores; además, se requiere claridad sobre programas de fertilización basados en la absorción diferencial de nutrientes en las diferentes etapas de desarrollo del cultivo; por estas consideraciones se realizó esta investigación con el objetivo de evaluar el contenido y distribución de macronutrientes ( $N, P, K$ ) en los órganos (tallos, hojas, flores y semillas) y en las diferentes etapas de desarrollo de la planta.

\section{Materiales y métodos}

La investigación se realizó en una pequeña explotación de la vereda la Olga, municipio de Yumbo, departamento de Valle del Cauca, Colombia, ubicado a $3^{\circ} 35^{\prime} 0^{\prime \prime} \mathrm{N}, 76^{\circ} 28^{\prime} 0^{\prime \prime} \mathrm{W}$, altitud entre 1650 y $2000 \mathrm{msnm}$, humedad relativa de $50 \%$ en época seca y $70 \%$ en época de lluvia.

El suelo experimental donde se estableció el cultivo de caléndula ( $C$. officinalis $L$.) fue caracterizado (física y química), tomando muestras de 0 a $20 \mathrm{~cm}$ de profundidad, las cuales fueron analizadas en el laboratorio de Física de Suelos de la Universidad Nacional de Colombia, Sede Palmira, utilizando para determinar: textura (método de la Pipeta de Robinson's), densidad aparente (método del cilindro biselado, Jaramillo, 2002), densidad real (método del picnómetro descrito por Montenegro \& Malagón (1990) y porosidad.

Así mismo, en el laboratorio de servicios analíticos del Centro Internacional de Agricultura Trópical (CIAT) se determinó: pH (1:2 en agua), carbono orgánico ( $\left.\mathrm{CO} \mathrm{g} \mathrm{kg}{ }^{-1}\right)$, fósforo (P-Bray II) $\left(\mathrm{mg} \mathrm{kg}^{-1}\right)$, potasio $(\mathrm{K})$, calcio $(\mathrm{Ca})$, magnesio $(\mathrm{Mg})$, sodio $(\mathrm{Na})$, aluminio(Al) y capacidad de intercambio catiónico (CIC) en $\mathrm{cmol} \mathrm{kg}^{-1}$; boro (B), azufre (S), cobre(Cu), hierro(Fe), manganeso(Mn) y zinc( $\mathrm{Zn})$ en $\mathrm{mg} \mathrm{kg}^{-1}$, 
de acuerdo a metodologías estandarizadas (CIAT, 2006). Los análisis químicos del suelo se interpretaron con las escalas del Instituto Geográfico Agustín Codazzi (IGAC, 2006) y Castro (2004).

El cultivo se manejó de acuerdo a la tecnología local de producción comercial y se definió como unidad de muestreo una parcela experimental de $1000 \mathrm{~m}^{2}$. Se utilizó un diseño completamente al azar, con cinco tratamientos y tres repeticiones. Se evaluó el contenido de macronutrientes en la planta completa y en cada órgano aéreo de la planta (hojas, tallos, flores y semillas), en cinco etapas fenológicas (2, 3, 5, 6 y 7 meses de edad después del trasplante (mdt)). En cada etapa se cosecharon 10 plantas al azar; las muestras de hojas, tallos, flores y semillas se lavaron con agua destilada, se secaron a $60^{\circ} \mathrm{C}$ hasta peso constante, se molieron utilizando un molino de cuchillas de tungsteno (IKA LABORTECHNIK M20), luego se tamizaron a $2 \mathrm{~mm}$, se marcaron y empacaron para trasladar al laboratorio.

Los análisis de contenido y distribución de N, P, y $\mathrm{K}$ en plantas completas y en hojas, tallos, flores y semillas, se realizaron en el laboratorio de servicios analíticos del CIAT por extracción mediante digestión ácida y determinación con auto analizador (espectrofotómetro automático).

Los datos fueron analizados mediante el paquete estadístico SAS (Statistical Analysis System) versión 9.13, con el cual se realizaron análisis de varianza y pruebas de comparación múltiple de promedios Duncan.

\section{Resultados y discusión}

En general las condiciones físicas y químicas del suelo permiten un desarrollo óptimo para el cultivo de caléndula; este suelo presentó textura franco limoso, lo que permite una adecuada laborabilidad (Tabla 1) siempre y cuando la humedad gravimétrica no supere el 50\% (Amézquita \& Londoño, 1997); además, presenta baja densidad aparente la cual favorece el buen crecimiento de raíces, drenaje y aireación (Jaramillo, 2002); alta porosidad (>50\%), adecuada para retener, transmitir agua y permitir el movimiento del aire.

Tabla 1. Caracterización física del suelo de la zona de estudio (vereda la Olga, Yumbo-Valle del Cauca).

\begin{tabular}{|c|c|c|c|c|c|c|c|}
\hline Profundidad & Arena & Limo & Arcilla & Clase Textural & $\begin{array}{l}\text { Densidad } \\
\text { aparente }\end{array}$ & $\begin{array}{l}\text { Densidad } \\
\text { real }\end{array}$ & $\begin{array}{c}\text { Porosidad } \\
\text { Total }\end{array}$ \\
\hline $\mathrm{cm}$ & $\%$ & $\%$ & $\%$ & & \multicolumn{2}{|c|}{$\mathrm{kg} \mathrm{m}^{-3}$} & $\%$ \\
\hline $0-20$ & 25,68 & 53,76 & 20,56 & Franco limoso & $0,83 \mathrm{~kg} \mathrm{~m}^{-3}$ & $2,54 \mathrm{~kg} \mathrm{~m}^{-3}$ & 67,32 \\
\hline
\end{tabular}

Fuente: Laboratorio de Física de Suelos UNAL Palmira.

El suelo presentó pH casi neutro, alto contenido de carbono orgánico, bajos niveles de $\mathrm{Fe}$ y $\mathrm{Cu}$, altos contenidos en $\mathrm{S}, \mathrm{B}, \mathrm{P}, \mathrm{Mn}, \mathrm{Ca}, \mathrm{Mg}, \mathrm{Na}$ y $\mathrm{Zn}$, alta $\mathrm{CIC}$, predominando la saturación por $\mathrm{Ca}$ y K (Tabla 2). Estos resultados indican que es un suelo con capacidad de brindar suficientes nutrientes durante las diferentes etapas de desarrollo de la caléndula. 
Tabla 2. Características químicas del suelo de la zona de estudio (vereda la Olga, Yumbo, Valle del Cauca)

\begin{tabular}{lccc}
\hline Determinaciones & Unidades & Valor & Nivel \\
\hline $\mathrm{pH}$ & & 6,92 & Acidez muy débil \\
$\mathrm{CO}$ & $\mathrm{g} \mathrm{kg-1}$ & 58,24 & Alto \\
$\mathrm{K}$ & & 7,9 & Alto \\
$\mathrm{Ca}$ & & 201,7 & Alto \\
$\mathrm{Mg}$ & $\mathrm{cmol}(\mathrm{c}) \mathrm{kg}-1$ & 33,5 & Alto \\
$\mathrm{Na}$ & & 2,2 & Alto \\
$\mathrm{CIC}$ & & 298 & Alto \\
$\mathrm{P}(\mathrm{Bray} \mathrm{II})$ & & 275,4 & Alto \\
$\mathrm{S}$ & & 60,03 & Alto \\
$\mathrm{B}$ & & 1,37 & Alto \\
$\mathrm{Fe}$ & & 1,21 & Bajo \\
$\mathrm{Mn}$ & $\mathrm{mg} \mathrm{kg}-1$ & 78,34 & Alto \\
$\mathrm{Cu}$ & & 0,35 & Bajo \\
$\mathrm{Zn}$ & & 17,36 & Alto \\
\hline
\end{tabular}

Fuente: Laboratorio de química de Suelos, CIAT.

Dinámica de macronutrientes en diferentes épocas de muestreo

Los contenidos de $\mathrm{N}$ presentaron diferencias altamente significativas en las épocas evaluadas, siendo los contenidos en los meses 2 y 5 similares estadísticamente, lo mismo que los meses 3 y 7 (Figura 1). Los mayores contenidos se registraron en el séptimo mes $\left(31,10 \mathrm{~g} \mathrm{~kg}^{-1}\right)$, seguido del tercero $\left(28,82 \mathrm{~g} \mathrm{~kg}^{-1}\right)$. En términos generales se observa una demanda de este nutriente en todas las épocas evaluadas, siendo necesaria su disponibilidad en todo el ciclo como lo expresa Rao (2009).
En cuanto al $\mathrm{P}$ el análisis de varianza mostró diferencias altamente significativas $(p<0.01)$ entre épocas evaluadas, siendo los meses dos y tres diferentes estadísticamente en relación con los demás (Figura 1). En las primeras etapas de desarrollo su disponibilidad es importante y los mayores contenidos se registran en el segundo y tercer mes (5,37 y $4,87 \mathrm{~g} \mathrm{~kg}^{-1}$ ), respectivamente, disminuyendo con la evolución del cultivo. Rao (2009) expresa que el P se requiere en las primeras etapas de crecimiento de la planta, ya que participa en procesos energéticos como la formación de ATP y ADP, entre otros. 

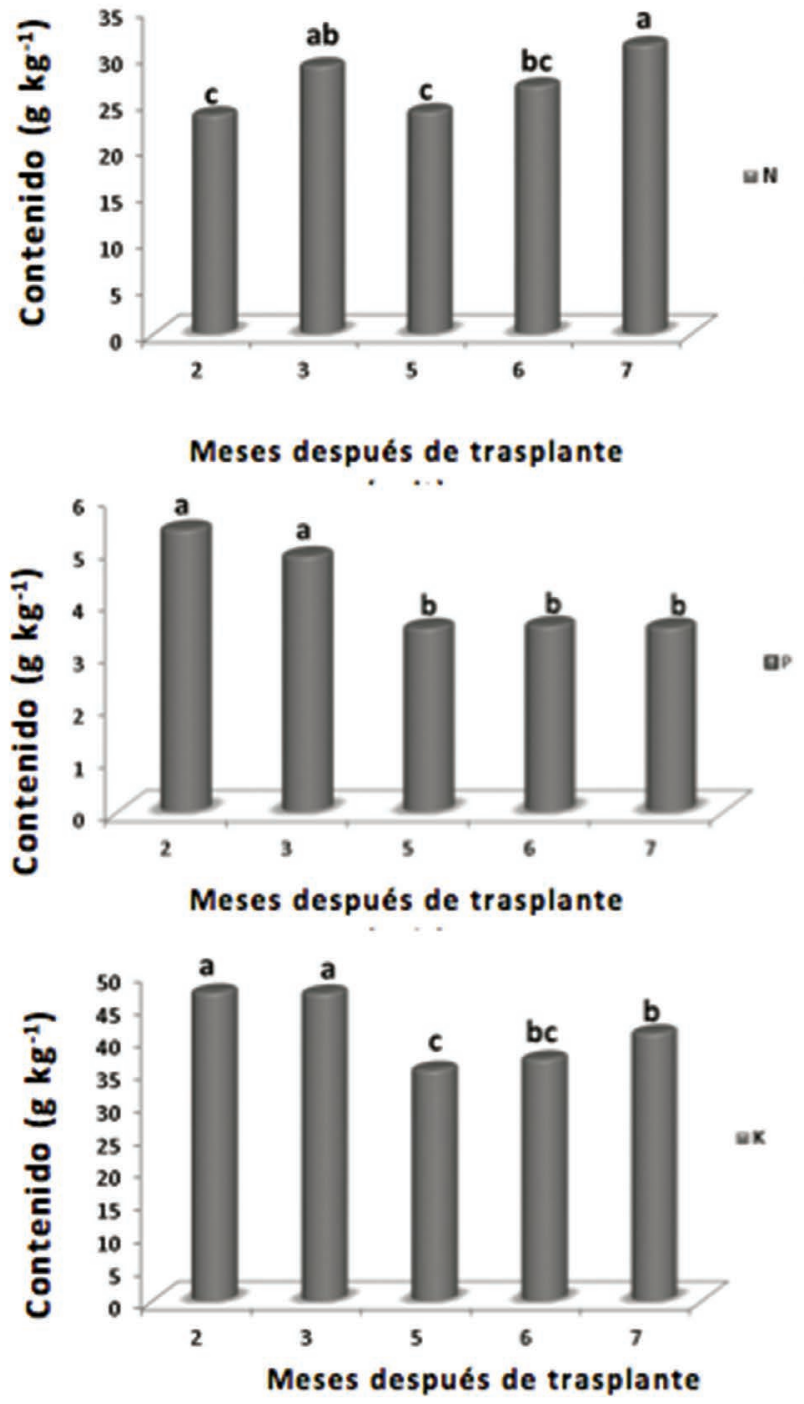

Figura 1. Contenidos de N, P, K en plantas de caléndula ( $C$. officinalis L.) en diferentes épocas de desarrollo. Promedios con igual letra no difieren significativamente $(p<0.05)$ según la prueba de Duncan

En cuanto a $\mathrm{K}$, se presentaron diferencias altamente significativas $(p<0.01)$ entre épocas, siendo los contenidos en los meses 2 y 3 , los más elevados (47,07 y 46,93 $\left.\mathrm{g} \mathrm{kg}^{-1}\right)$, similares estadísticamente y diferentes a los demás meses (Figura 1). El potasio mantiene una dinámica de alta demanda como lo menciona Rao (2009), debido a su participación en la síntesis y transporte de fotosintatos a los órganos de reserva, como también, en la producción de carbohidratos, proteínas, aceites, etc. (Tisdale, Haylin \& Nelson, 2005), así, se considera este elemento de vital importancia para la planta, ya que participa en casi todos sus procesos fisiológicos (Valencia, 1999), incluyendo procesos de osmoregulación como mecanismo adaptativo a condiciones de estrés hídrico (Marschner, 2003).

Cuando se evalúa el consumo de nutrientes en las diferentes épocas, se observa que el $\mathrm{K}$ fue el de mayor demanda (197, $\left.40 \mathrm{~g} \mathrm{~kg}^{-1}\right)$, seguido del $\mathrm{N}\left(130,53 \mathrm{~g} \mathrm{~kg}^{-1}\right)$ y $\mathrm{P}\left(21,19 \mathrm{~g} \mathrm{~kg}^{-1}\right)$, respectivamente. Resultados similares fueron reportados por Teixeira, Polato \& Tavares (2000), concluyendo que los macronutrientes $\mathrm{N}$ y $\mathrm{K}$ fueron los más exigidos por la caléndula bajo condiciones controladas; el $\mathrm{N}$ fue el segundo macronutriente más acumulado, coincidiendo con lo reportado por Filgueira (2003) quien afirma que éste es normalmente el segundo nutriente más requerido por plantaciones olerícolas después del K. Por otro lado, en experimentos realizados por Król (2011), demostró que la caléndula exige cantidades considerables de $\mathrm{N}$, sin embargo, a partir de $80 \mathrm{~kg} \mathrm{~N}^{-1}$ no se observó mayor productividad.

La absorción del $\mathrm{P}$ es restringida por factores, en este caso de elevadas concentraciones de $\mathrm{Ca}$ y $\mathrm{Mg}$, como lo muestra el análisis de suelos realizado, es posible que este nutriente forme compuestos insolubles con los cationes mencionados y por tanto no esté disponible para la planta.

\section{Dinámica de macronutrientes en órganos de la planta para diferentes etapas de desarrollo}

Nitrógeno. Se detectaron diferencias altamente significativas $(p<0.01)$ en el contenido de macronutrientes en los órganos evaluados (Figura 2). Los mayores contenidos se localizaron en las flores $\left(32,33 \mathrm{~g} \mathrm{~kg}^{-1}\right)$ y hojas $\left(29,0 \mathrm{~g} \mathrm{~kg}^{-1}\right)$ y, los más bajos en los tallos $\left(19,09 \mathrm{~g} \mathrm{~kg}^{-1}\right)$ y las semillas $(21,87 \mathrm{~g}$ $\left.\mathrm{kg}^{-1}\right)$. Durante las épocas se aprecia un comportamiento fluctuante en flores y tallos, mientras que en las hojas tiende a elevar su contenido con el transcurso del tiempo, a diferencia de las semillas en las cuales decrece. 

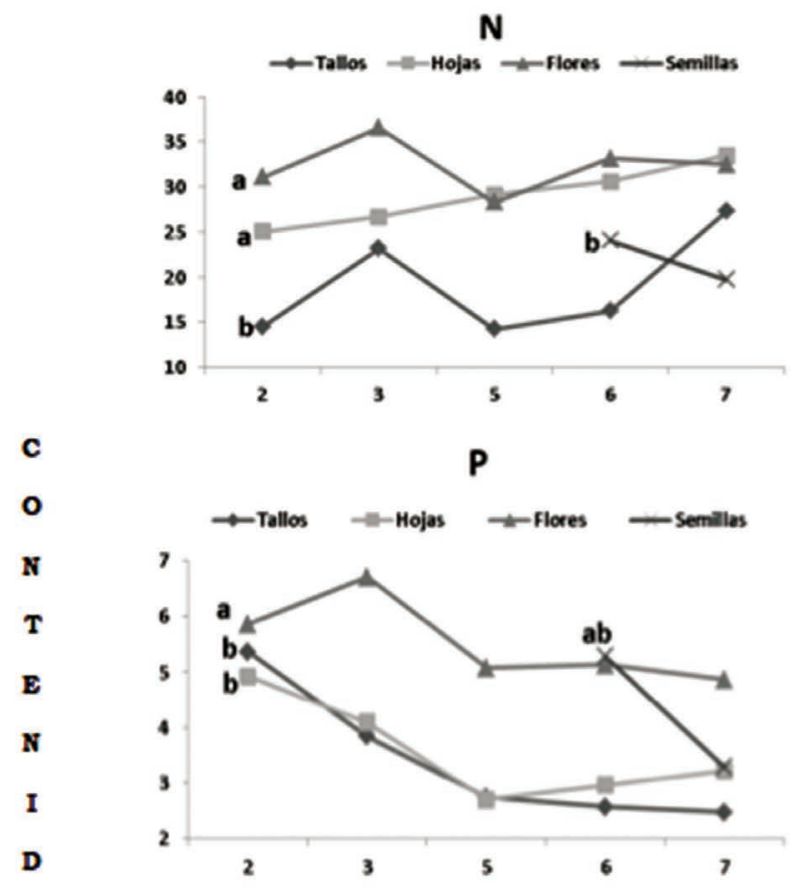

0

$\mathbf{~ k g}^{\mathbf{2}}$

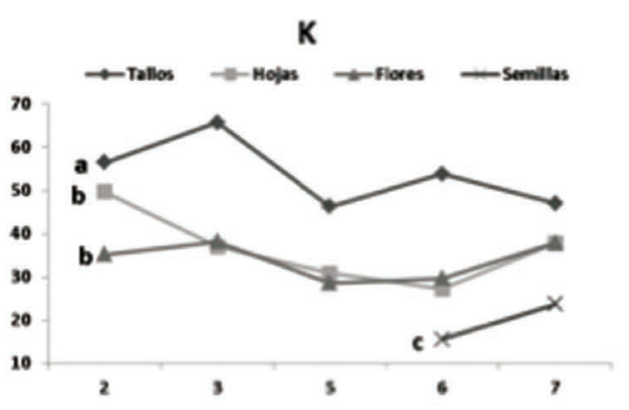

Etapas de muestreo

Figura 2. Contenido de macronutrientes en tallos, hojas, flores y semillas de la planta de caléndula en diferentes etapas fenológicas. Promedios con igual letra no difieren significativamente $(p>0.05)$ según

la Prueba de Duncan.

Fósforo. El Análisis de varianza mostró diferencias significativas $(p<0.05)$ para el contenido de $P$ entre los diferentes órganos, como lo sugieren Puentes et al (2014) afirmando que el contenido de fósforo es diferente en hojas y semillas. Así, el contenido en las flores y semillas no difirieron estadísticamente ( $p>0.05)$ al igual que en el contenido de fósforo en tallos y hojas (Figura 2). La mayor concentración de $P$ se registró en flores $(5,52 \mathrm{~g}$ $\left.\mathrm{kg}^{-1}\right)$ y semillas $\left(4,27 \mathrm{~g} \mathrm{~kg}^{-1}\right)$, coincidiendo con los resultados obtenidos por Moreira et al. (2005) y Puentes, Menjivar, Gómez \& Aranzazu (2014). La concentración menor se presentó en tallos y hojas (3,39 y $3,58 \mathrm{~g} \mathrm{~kg}^{-1}$, respectivamente).

Al inicio del ciclo del cultivo, el fósforo fue el nutrimento requerido en mayores cantidades, cuyas funciones energéticas son necesarias en muchos procesos fisiológicos y metabólicos, participando entre otros en la formación de raíces (Marschner, 2003). Con el trascurrir del tiempo su demanda disminuye, localizándose principalmente en flores y semillas órganos responsables de la supervivencia de la planta (Rao, 2009). En caléndula se encontraron contenidos de fósforo en rangos de 2 a $6 \mathrm{~g} \mathrm{~kg}^{-1}$, en general con los valores mayores en las primeras etapas del cultivo (segundo y tercer mes). Posiblemente estos contenidos están relacionados con el fenómeno de dilución de nutrientes que se presenta cuando la planta adquiere mucha más biomasa en el proceso normal de crecimiento, ya que los contenidos en los meses próximos a la senescencia de la planta son menores (Malavolta, Vitti \& Oliveira, 1989).

Potasio. Se detectaron diferencias altamente significativas $(p<0.01)$ entre los órganos para el contenido de este macronutriente. El contenido en el tallo $\left(53,82 \mathrm{~g} \mathrm{~kg}^{-1}\right)$ fue diferente estadísticamente de los demás, siendo el contenido en las hojas y flores similares entre sí (Figura 2). El K fue el elemento de mayor contenido en todo el ciclo y su importancia radica en ser cofactor de más de 40 enzimas y regulador de turgencia celular (Mengel \& Kirkby, 2000). En maíz y otros cereales en los tejidos del tallo se presentan los contenidos mayores y en su ausencia la planta se cae con facilidad y susceptible al ataque de plagas en las raíces (Rao, 2009).

La absorción y extracción de nutrientes de un cultivo permite conocer la cantidad requerida para producir un rendimiento dado en un periodo de tiempo definido (Bertsch, 2003); en ese orden de ideas los resultados de esta investigación permiten que los productores puedan elaborar un plan de fertilización basado en la extracción y absorción de nutrientes y satisfacer las necesidades del cultivo. 


\section{Conclusiones}

Las épocas de aplicación de fertilizantes deben orientarse teniendo en cuenta el contenido de nutrientes del suelo y la demanda de nutrientes por la planta en cada etapa de desarrollo; en este sentido, para el cultivo de caléndula (C. officinalis L.) se debe dar importancia a la fertilización con NPK en los tres primeros meses del cultivo.

El contenido de NPK en los diferentes órganos de la planta evaluados, evidencia una concentración diferencial de nutrientes, así, el cultivo presenta mayor concentración de $\mathrm{N}$ y $\mathrm{P}$ en flores, y de $\mathrm{K}$ en los tallos.

\section{Agradecimientos}

Los autores agradecen a la Universidad Nacional de Colombia, sede Palmira, a la Dirección de Investigación (DIPAL), por la financiación parcial de la investigación, al grupo de investigación "Recursos genéticos de plantas medicinales, aromáticas y condimentarías" y al grupo "Uso y manejo de suelos y aguas con énfasis en degradación de suelos". Al Dr. Diosdado Baena G. por su asesoría estadística, a los agricultores del corregimiento Los cerros de La Olga por su constante colaboración, y a los funcionarios del Laboratorio de Servicios Analíticos del CIAT.

\section{Literatura citada}

1. Acosta de la L., L., Rodríguez, F.C., \& Sánchez G. E. (2001). Instructivo técnico de Calendula officinalis. Revista Cubana de Plantas Medicinales 6(1): 23-27. La Habana, Cuba: Ciencia médicas.

2. Alarcón, A. (2000). Tecnología para cultivos de alto rendimiento. Murcia, España: Novedades Agrícolas.

3. Amézquita, E. \& Londoño, H. (1997). La infiltración del agua en algunos suelos de los Llanos Orientales y sus implicaciones en su uso y manejo. Revista Suelos Ecuatoriales Vol.27:163-168. Medellín, Colombia: Sociedad Colombiana de la Ciencia del Suelo.

4. Araujo, C.B.O., Santos, A.M., Fernandes, L.A., Martins, E.R., Sampaio, R.A., Costa, C.A. \& Leite, G.L.D. (2009).
Uso da adubação orgãnica e cobertura morta na cultura da Calêndula (Calendula officinalis L.). Rev. Bras. Plantas Med. (11): 117-123. Paulínia, Brasil: Sociedade Brasileira.

5. Bertsch, F. (2003). Absorción de nutrimentos por los cultivos. San José, Costa Rica. Asociación Costarricense de la Ciencia del Suelo.

6. Borella, J.C., Ribeiro, N.S., Freato, A.M.R., Mazzo, K.F. \& Barbosa. D.M. D.M. (2011). Influencia da adubação e da cobertura morta na produtividade e no teor de flavonoides de Calendula officinalis L. (Asteraceae). Rev. Bras. Plantas Med. (13): 235-239. Paulínia, Brasil: Sociedade Brasileira.

7. Cáceres, A. (1996). Plantas de uso medicinal en Guatemala. Univesidad San Carlos de Guatemala. Ciudad de Guatemala, Guatemala: Editorial Universitaria.

8. Castro, H. (2004). Propuesta guía de indicadores analíticos para calificar suelos estables y en proceso de degradación desde el punto de vista físico. En el Centro Internacional de Agricultura Tropical, Taller Nacional sobre indicadores de calidad del suelo, llevado a cabo en Palmira, Colombia.

9. Castro, H.E. \& Gómez, M.I. (2010). Fertilidad del Suelo y Fertilizantes. En Burbano H.F. Silva (ed), Ciencia del suelo, Principios básicos (pp.105-130) Bogotá, D.C., Colombia: Sociedad Colombiana de la ciencia del suelo.

10. CIAT (Centro Internacional de Agricultura Tropical). (2006). Manual de análisis de suelos y tejido vegetal. Cali, Colombia: CIAT.

11. Conpes 3514. (2008). Consejo Nacional de Política Económica y Social República de Colombia, Bogotá D.C., Colombia: Conpes.

12. Fernandes, E. F. A., Meloni, F., Borella, J. C. \& Lopes, N. P. (2013). Effect of fertilization and harvest period on polar metabolites of Calendula officcinalis. Revista Brasileira de Farmacognosia. 23(5):731-735. Paraná, Brasil: Brazilian Society of Pharmacognosy.

13. Filgueira, F.A.R. (2003). Novo manual de olericultura: Agrotecnologia moderna na produção e comercialização de hortaliças. 2ed. Viçosa, Brasil: UFV.

14. García, B. (1975). Flora Medicinal de Colombia. Bogotá D.C, Colombia: Universidad Nacional de Colombia.

15. Gonçalves, A. C., Jacobus de M, A., Lindino, C. A., Nacke, H. \& Antonio de C. E. (2012). Availability of nutrients and toxic heavy metals in marigold plants. Acta Scientiarum Technology 34 (4): 451-456. Maringá-Eduem, Brasil: Universidade Estadual de Maringá-Eduem.

16. Guevara, H. A., Luengas, P. E. \& Garavito, G. (2010). Revisión documental de los productos naturales legalmente autorizados para su mercadeo en Colombia. Colombia Médica 41(2): 129-140. Cali, Colombia: Universidad del Valle.

17. IGAC (Instituto Geográfico Agustín Codazzi). (2006). Métodos analíticos del Laboratorio de Suelos. Subdirección Agrológica, 4a ed. Bogotá D.C, Colombia: IGAC.

18. Jaramillo, D. (2002). Introducción a la Ciencia del Suelo. Medellin, Colombia: Universidad Nacional de Colombia

19. Król, B. (2011). Yield and The Chemical Composition of 
Flower Heads of Pot Marigold Calendula officinalis L. cv. Orange King Depending on Nitrogen Fertilization. Acta Sci. Pol. Hortorum Cultus. 10(2):235-243. Lublin, Polonia:University of Life Science in Lublin

20. Malavolta, E., Vitti, G.C. \& Oliveira, A.S. (1989). Avaliação do estado nutricional das plantas: princípios e aplicações. Piracicaba, Brasil: Associação Brasileira para Pesquisa da Potassa e do Fosfato.

21. Marschner, H. (2003). Mineral Nutrition of Higher Plants. 2nd edition...London, Reino Unido: Academic Press.

22. Mengel, K. \& Kirkby, E. (2000). Principios de nutrición vegetal. Traducción al español de la 4a edición de Melgar, R. J. y Ruíz, M. (1987). Basel, Switzerland: International Potash Institute.

23. Mincomex (Ministerio de Comercio Exterior) (2009). "Sector de cosméticos y productos de aseo - Desarrollando sectores de talla mundial en Colombia." Bogota D.C, Colombia: Ministerio de Comercio, Industria y Turismo.

24. Montenegro, H. \& Malagón, C. (1990). Propiedades físicas de los suelos. Ministerio de Hacienda y Crédito Público. Bogotá D.C. Colombia: Instituto Geográfico "Agustín Codazzi".

25. Moreira, P. A., Marchetti, M. E., Vieira, M. C., Novelino, J. O., Gonçalves, M. C. \& Robaina, A. (2005). Desenvolvimento vegetativo e teor foliar de macronutrientes da calêndula (Caléndula officinalis L.) adubada com nitrogênio e fósforo. Rev. Bras. Pl. Med. Botucatu 8 (1):1823. Paulinia, Brasil: Unicamp

26. Paim, L.F.M.A., Fontana, M., Winckler, M., Grando, A.P.,
Muneron, T.L. \& Roman Jr., W.A. (2010). Assessment of plant development, morphology and flavonoid content in different cultivation treatments of Calendula officinalis L. Asteraceae. Rev. Bras. Farmacogn. 20: 974-980. Paraná, Brasil: Universidade federal do Paraná

27. Pérez, A.E. (1956). Plantas útiles de Colombia. 3ra Edition. Madrid, España: Rivadeneyra

28. Puentes, Y.J., Menjivar, J.C., Gómez, A. \& Aranzazu, F. (2014). Absorción y distribución de nutrientes en clones de cacao y sus efectos en el rendimiento. Acta Agronómica, 63(2): 145-152. Palmira, Valle del Cauca: Universidad Nacional de Colombia

29. Rao, I.M. (2009). Essential plant nutrients and their functions. Working Document No. 36. Cali, Colombia: Centro Internacional de Agricultura Tropical (CIAT)

30. Sánchez, M. E. (1980). Diccionario de plantas agrícolas. Servicio de Publicaciones Agrarias: Madrid, España: Ministerio de Agricultura.

31. Teixeira, N.T., Polato, A.M. \& Tavares, M.A.G.C. (2000). Extração de nutrientes pela cultura da caléndula ( $\mathrm{Ca}$ lendula officinalis L.). Rev. Ecosistema Vol. 25(2):135136. Espirito Santo, Brasil: Centro Regional Universitario de Espirito Santo do pinhal

32. Tisdale, S.L., Havlin, J. \& Nelson, W. (2005). Fertility and fertilizers An Introduction to nutrient Management. Seventh edition. New Jersey, E.E.U.U: Prentice Hall

33. Valencia, A.G. (1999). Fisiología, nutrición y fertilización del cafeto. Chinchiná (Colombia), Agro insumos del café S.A. Chinchina, Manizales: Cenicafé

\section{Conflicto de Intereses}

Los autores declaran no tener ningún conflicto de intereses 
UNAP 